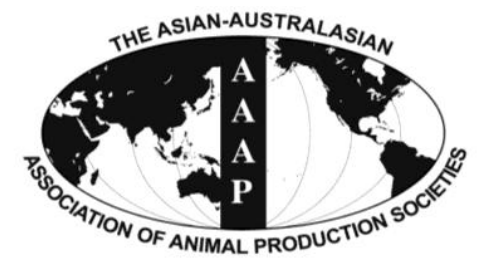

Asian Australas. J. Anim. Sci.

Vol. 26, No. 12 : 1742-1752 December 2013

http://dx.doi.org/10.5713/ajas.2013.13266

www.ajas.info

pISSN 1011-2367 elSSN 1976-5517

\title{
Growth Performance, Carcass Traits and Serum Mineral Chemistry as Affected by Dietary Sodium and Sodium Salts Fed to Broiler Chickens Reared under Phase Feeding System
}

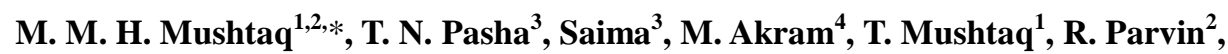 \\ U. Farooq ${ }^{5}$, S. Mehmood ${ }^{4}$, K. J. Iqbal ${ }^{6}$, and J. Hwangbo ${ }^{2}$ \\ Department of Poultry Production, University of Veterinary and Animal Sciences, Lahore, 54000, Pakistan
}

\begin{abstract}
A basal diet $(0.8 \mathrm{~g} / \mathrm{kg} \mathrm{dNa})$ was formulated in which each of the two sources $\left(\mathrm{NaHCO}_{3}\right.$ and $\left.\mathrm{Na}_{2} \mathrm{SO}_{4}\right)$ were supplemented in such a way to attain four levels $(1.7,2.6,3.5$, and $4.4 \mathrm{~g} / \mathrm{kg}$ ) of total $\mathrm{dNa}$, respectively, under $4 \times 2$ factorial arrangement. Eight dietary treatments were replicated four times, with 40 birds in each replicate $(n=1,280)$. The diets supplemented with $\mathrm{Na}_{2} \mathrm{SO}_{4}$ to attain higher levels of dNa showed highest BW gain and feed intake (FI) during d 1 to 10 (interaction effects) while $2.6 \mathrm{~g} / \mathrm{kg} \mathrm{dNa}$ exhibited improved BW gain and gain:feed (FG) during d 11 to 20. Linear rise in daily water intake (DWI) was associated with diets containing increasing dNa during d 1 to 42 ( $\leq \leq 0.036)$. During the first 10 d, DWI:FI was found highest in $\mathrm{NaHCO}_{3}$ diets while $\mathrm{Na}_{2} \mathrm{SO}_{4}$ diets showed highest DWI:FI during last $10 \mathrm{~d}$ of the experiment $(\mathrm{p} \leq 0.036)$. Increasing $\mathrm{dNa}$ and changing $\mathrm{Na}_{2} \mathrm{SO}_{4}$ with $\mathrm{NaHCO}_{3}$ salt increased $\mathrm{pH}$ and resulted in poor growth performance. Dressing weight $(\mathrm{p} \leq 0.001)$ and abdominal fat $(\mathrm{p} \leq 0.001$; quadratic effect) were reduced, whereas breast $(\mathrm{p} \leq 0.001)$ and thigh $(\mathrm{p}<0.001)$ weights were aggravated with increasing dNa (linear effects). Present findings suggested higher levels of $\mathrm{dNa}$ from $\mathrm{Na}_{2} \mathrm{SO}_{4}$ as the supplemental salt in broiler diets would produce better growth performance, especially in first ten days of life, and improve carcass and body organ characteristics. (Key Words: Broiler, Growth and Carcass Responses, Phase Feeding Program, Serum Mineral Chemistry, Sodium)
\end{abstract}

\section{INTRODUCTION}

Sodium (Na), the principal cation of extracellular fluid, is known to involve in a number of physiological functions like regulation of extracellular fluid volume, acid base balance, and membrane potential of cells. The concentration of $\mathrm{Na}$ in the extracellular fluid is maintained through

\footnotetext{
* Corresponding Author: M. M. H. Mushtaq. Tel: +82-41-5806728, Fax: +82-41-580-6719, E-mail: haroon@korea.kr

${ }^{1}$ AgroVisions, Faisalabad, 38800, Pakistan.

${ }^{2}$ Poultry Science Division, National Institute of Animal Science, 114, Sinbang 1-gil, Seonghwan-eup, Seobuk-gu, Cheonan-si, 331801, Korea.

${ }^{3}$ Department of Animal Nutrition, University of Veterinary and Animal Sciences, Lahore, 54000, Pakistan.

${ }^{4}$ Department of Poultry Production, University of Veterinary and Animal Sciences, Lahore, 54000, Pakistan.

${ }^{5}$ School of Animal Biology, University of Western Australia, 35 Stirling Hwy, Crawley WA 6009, Australia.

${ }^{6}$ Department of Fisheries and Aquaculture, University of Veterinary and Animal Sciences, Lahore, 54000, Pakistan.

Submitted May 15, 2013; Accepted Jul. 11, 2013; Revised Aug. 8, 2013
}

$\mathrm{Na}^{+} / \mathrm{K}^{+}$-ATPase pump, which expels $\mathrm{Na}$ from the cell (Leeson and Summers, 2001). It is anticipated that the addition of dietary cations $(\mathrm{Na} / \mathrm{K})$ could be used to compensate depressed growth in chickens caused by high levels of dietary anions $\left(\mathrm{Cl} / \mathrm{HCO}_{3}\right)$ and vice versa. In our previous study, we observed better weight gain and gain:feed in $0.31 \%$ chloride diets supplemented with $0.30 \%$ $\mathrm{Na}$ (Mushtaq et al., 2013).

Mongin (1980) described the effect and interrelationship of $\mathrm{Na}, \mathrm{K}$ and $\mathrm{Cl}$ into an equation, called dietary electrolyte balance (DEB $\leq \mathrm{Na}+\mathrm{K}-\mathrm{Cl}, \mathrm{mEq} / \mathrm{kg}$ diet), and different researchers (Murakami et al., 2000, 2001; Rondon et al., 2001; Borges et al., 2003a, 2003b, 2004a,; 2004b; Mushtaq et al., 2005, 2007; Ahmad et al., 2005, 2009) recommended different DEB for broilers at different ages, ranging from 150 to $350 \mathrm{mEq} / \mathrm{kg}$ with various combinations of dietary $\mathrm{Na}$ (dNa), K and Cl. Mushtaq et al. (2005 and 2007) reported that different ions acted differently on a similar DEB of 250 $\mathrm{mEq} / \mathrm{kg}$.

The supplementation of various salts is anticipated to change the bird's osmotic balance and this change is mainly 
influenced by the contributing electrolytes and ultimately leads to alter water consumption and excretion (Smith and Teeter, 1989; Borges et al., 2004a, 2004b). Sodium bicarbonate $\left(\mathrm{NaHCO}_{3}\right)$ is considered as the first-rated supplemental salt as a source of $\mathrm{Na}$ and $\mathrm{HCO}_{3}$ when compared with other salts (Johnson and Karunajeewa, 1985; Gorman and Balnave, 1994; Ahmad et al., 2005). Sodium sulphate $\left(\mathrm{Na}_{2} \mathrm{SO}_{4}\right)$ is known to induce blood acidosis with great severity when compared with other sulphate sources hence acidic properties of sulphates is directly linked with the supplemental salt (Ruiz-Lopez and Austic, 1993; Ahmad et al., 2005). Genetics and nutrient requirements of today's broilers are changing with respect to varying growing potential on a daily basis, hence there is a need to reconsider the requirements of $\mathrm{dNa}, \mathrm{DEB}$ and sodium salts for broilers raised under phase feeding.

The present study was, therefore, envisaged to evaluate the effect of supplementation of $\mathrm{dNa}$ with the applicability of the DEB using different sodium salts on growth and carcass traits, body physiological responses, water intake, litter condition and serum mineral chemistry of the modern broiler strain fed under the phase feeding program.

\section{MATERIALS AND METHODS}

All the experimental procedure was approved by Advanced Study and Research Board, University of Veterinary and Animal Sciences, Lahore-Pakistan.

\section{Housing and management}

A total of 1,280 one-d-old $(42.1 \pm 0.8 \mathrm{~g})$ straight-run Hubbard broiler chicks (Hubbard $\times$ Hubbard) were randomly allocated to eight dietary treatments replicated four times in such a way that each replicate had 40 birds. Each replicate pen was equipped with separate overhead, transparent and volume-graduated twenty liters $(20 \mathrm{~L})$ water bottles linked to nipple drinker line. Water bottles were cleaned and filled with fresh water after measuring the water consumption on a daily basis. One flat bottom round feeder was provided for each experimental pen. Birds were housed in environmentally controlled systems where variation in temperature and relative humidity were recorded and maintained according to the production manual. Continuous light was provided $24 \mathrm{~h}$ for first $3 \mathrm{~d}$ and then 23L:1D light pattern was adopted for the rest of the experimental period. A $7.5 \mathrm{~cm}$ deep fresh sawdust was used as litter material over a concrete floor. For the first $3 \mathrm{~d}$, house temperature was maintained at $32^{\circ} \mathrm{C}$ and thereafter reduced by $0.5^{\circ} \mathrm{C}$ per day until $24^{\circ} \mathrm{C}$ was attained at $\mathrm{d} 19$.

Birds were vaccinated against Newcastle Disease (ND) plus Infectious Bronchitis viruses at $4 \mathrm{~d}$, Infectious Bursal Disease virus at $8 \mathrm{~d}$ and again at $14 \mathrm{~d}$; Hydropericardium
Syndrome virus at $18 \mathrm{~d}$ and ND-Lasota strain at $22 \mathrm{~d}$ following the locally designed vaccination schedule.

\section{Dietary plan}

A basal diet having $\mathrm{dNa}, \mathrm{K}$ and $\mathrm{Cl}$ as $0.8,7.1$ and 2.0 $\mathrm{g} / \mathrm{kg}$, respectively with a DEB value of $160 \mathrm{mEq} / \mathrm{kg}$ (Table 1) was formulated on least-cost basis. Afterwards, four levels of $\mathrm{dNa}(1.7,2.6,3.5$ and $4.4 \mathrm{~g} / \mathrm{kg})$ were attained from the basal diet $(0.8 \mathrm{~g} / \mathrm{kg})$ by using either feed-grade $\mathrm{NaHCO}_{3}$ or $\mathrm{Na}_{2} \mathrm{SO}_{4}$, which corresponded to DEB values of 200, 240, 280 and $320 \mathrm{mEq} / \mathrm{kg}$, respectively. For this purpose, a large batch of basal diet was prepared for each phase and then each of the experimental diets (i.e. eight) was prepared according to research plan using the basal diet. The experimental period was divided into four phases i.e., prestarter (d 1 to 10 ), starter (d 11 to 20 ), grower (d 21 to 33 ) and finisher (d 34 to 42), which met or exceeded the nutrient specifications recommended by the Hubbard management guide (Hubbard, 2004; Table 1).

\section{Dietary analyses}

All the ingredients were assayed for their proximate composition (AOAC, 2005) prior to diet formulation and actual values were used in the formulation. The $\mathrm{Na}$ and $\mathrm{K}$ contents of each diet were analyzed by flame photometer (AOAC, 2005) and $\mathrm{Cl}$ by titration with silver nitrate (Lacroix et al., 1970). The $\mathrm{Na}, \mathrm{K}$ and $\mathrm{Cl}$ contents of the final diets were again verified prior to start of the experiment. The ME of each ingredient was calculated by the appropriate regression equation suggested by the NRC (1994). Amino acid composition of each ingredient was calculated using AminoDat 3.0 Platinum (Degussa AG Feed Additives, 2006) based on the DM and CP contents of each ingredient. The amino acid composition of each diet met or exceeded the ideal amino acid ratio suggested by Han and Baker (1994). The experiment lasted for $42 \mathrm{~d}$ of age, offering mash diets throughout the experimental period.

\section{Live performance}

Data on feed intake (FI), BW gain (BWG) and feed-togain ratio (FG) was recorded for each phase. The feed was withheld for $6 \mathrm{~h}$ before weighing the birds at the end of each phase to ensure the emptying of the digestive tract of the bird (Table 2). Mortality was recorded on a daily basis and dead bird was weighed prior to removal to correct FG.

\section{Water intake}

Daily Water intake (DWI) was recorded from each replicate and a ratio between DWI and FI (DWI:FI) was also calculated for each phase (Table 3 ).

\section{Litter moisture}

Litter was collected at the end of each phase to 
Table 1. Ingredient and nutrient composition of the basal diets fed four levels of sodium with two sources of sodium salts at different stages of growth in broilers ${ }^{1}$

\begin{tabular}{|c|c|c|c|c|}
\hline & $\begin{array}{l}\text { Pre-starter } \\
\text { (d } 1 \text { to } 10 \text { ) }\end{array}$ & $\begin{array}{c}\text { Starter } \\
\text { (d } 11 \text { to 20) }\end{array}$ & $\begin{array}{c}\text { Grower } \\
\text { (d } 21 \text { to33) }\end{array}$ & $\begin{array}{l}\text { Finisher } \\
\text { (d } 34 \text { to 42) }\end{array}$ \\
\hline \multicolumn{5}{|l|}{ Ingredients $(\mathrm{g} / \mathrm{kg})$} \\
\hline Corn & 469.3 & 475.4 & 674.6 & 679.4 \\
\hline Broken rice & 134.6 & 161.6 & 0.7 & - \\
\hline Soybean meal (CP: 44.1\%) & 276.3 & 291.1 & 269.3 & 244.7 \\
\hline Canola meal (CP: 37.3\%) & 63.9 & 12.4 & - & - \\
\hline Oil & 16.2 & 22.1 & 18.8 & 39.4 \\
\hline Dicalcium phosphate & 21.6 & 20.2 & 18.9 & 16.0 \\
\hline Limestone & 10.4 & 9.4 & 10.5 & 11.0 \\
\hline L-lysine $\mathrm{HCl}$ & 2.3 & 2.2 & 2.0 & - \\
\hline L-lysine sulphate & - & - & - & 3.4 \\
\hline $\mathrm{NaCl}$ & 1.5 & 1.6 & 1.6 & 1.6 \\
\hline $\mathrm{KCl}$ & 0.4 & 0.3 & 0.5 & 1.4 \\
\hline DL-methionine & 2.0 & 2.0 & 1.7 & 1.7 \\
\hline L-threonine & 0.5 & 0.7 & 0.3 & 0.4 \\
\hline Premix $^{2}$ & 1.0 & 1.0 & 1.0 & 1.0 \\
\hline \multicolumn{5}{|c|}{ Nutrients (g/kg or otherwise stated; Analysis results) } \\
\hline $\operatorname{ME}(\mathrm{kcal} / \mathrm{kg})^{3}$ & 2,903 & 3,010 & 3,102 & 3,155 \\
\hline Crude protein & 211.0 & 202.0 & 189.0 & 182.0 \\
\hline Calcium & 10.0 & 9.0 & 9.0 & 8.5 \\
\hline Available Phos. & 4.5 & 4.2 & 4.0 & 3.5 \\
\hline Sodium & 0.8 & 0.8 & 0.8 & 0.8 \\
\hline Potassium & 7.1 & 7.1 & 7.1 & 7.1 \\
\hline Chloride & 2.0 & 2.0 & 2.0 & 2.0 \\
\hline DEB $(\mathrm{mEq} / \mathrm{kg})^{4}$ & 160 & 160 & 160 & 160 \\
\hline Dig lys & 11.0 & 10.5 & 9.7 & 9.3 \\
\hline
\end{tabular}

${ }^{1}$ All the diets were supplemented with 4 levels of either $\mathrm{NaHCO}_{3}\left(3.3,6.6,9.9\right.$, or $13.2 \mathrm{~g} / \mathrm{kg}$ ) or $\mathrm{Na}_{2} \mathrm{SO}_{4}(2.8,5.6,8.4$, or $11.2 \mathrm{~g} / \mathrm{kg})$ to make final dNa concentrations of $1.7,2.6,3.5$, or $4.4 \mathrm{~g} / \mathrm{kg}$, respectively. The basal diet has $0.8 \mathrm{~g} / \mathrm{kg} \mathrm{dNa}$ in it.

${ }^{2}$ Provides per $\mathrm{kg}$ of finished diet: vitamin $\mathrm{A}, 12 \mathrm{mg}$; vitamin $\mathrm{D}_{3}, 7 \mathrm{mg}$; vitamin E, $100 \mathrm{mg}$; vitamin $\mathrm{K}_{3}(50 \%$ as menadione nicotinamide bisulfite), $3 \mathrm{mg}$; vitamin $\mathrm{B}_{1}(98 \%), 3 \mathrm{mg}$; vitamin $\mathrm{B}_{2}(800,000 \mathrm{mg}), 12 \mathrm{mg}$; vitamin $\mathrm{B}_{3}$ (niacin; 99\%), $600 \mathrm{mg}$; vitamin $\mathrm{B}_{6}(98 \%), 4 \mathrm{mg}$; vitamin $\mathrm{B}_{9}$ (folic acid; $\left.95 \%\right), 2$ $\mathrm{mg}$; vitamin $\mathrm{B}_{12}(0.10 \%), 20 \mathrm{mg}$; Biotin $(0.10 \%), 5 \mathrm{mg}$; Ca-Pentothenate (98\%), $12 \mathrm{mg}$; choline (70\% as choline sodium), $1 \mathrm{~g}$; $\mathrm{MnO}(60 \%), 169 \mathrm{mg}$; $\mathrm{FeSO}_{4}(21 \%), 200 \mathrm{mg} ; \mathrm{ZnSO}_{4}$ (36\%), $150 \mathrm{mg} ; \mathrm{CuSO}_{4}$ (25\%), $40 \mathrm{mg}$; Se (sodium selenite 0.40\%), $100 \mathrm{mg}$; KI (68\%), $2 \mathrm{mg}$; Salinomycin, $60 \mathrm{mg}$; Zinc bacitracin (as Albac 10\%), $50 \mathrm{mg}$.

${ }^{3}$ Calculated values (NRC, 1994). ${ }^{4}$ Dietary electrolyte balance $=(\% \mathrm{Na} \times 10,000 / 23)+(\% \mathrm{~K} \times 10,000 / 39.1)-(\% \mathrm{Cl} \times 10,000 / 35.5)$.

determine its moisture (LM). For this purpose, about $500 \mathrm{~g}$ litter sample was randomly collected from different locations in each replicate pen. Each sample was homogenized and a representative sample of $100 \mathrm{~g}$ was taken and oven dried at $105^{\circ} \mathrm{C}$ for $24 \mathrm{~h}$ (AOAC, 2005) to determine moisture contents.

\section{Water analysis}

Water characteristics were also recorded twice (morning and noon) daily to check $\mathrm{pH}$ by $\mathrm{pH}$ metre (LT-Lutron $\mathrm{pH}$ 207 Taiwan), dissolved oxygen (DO) by DO metre (YSI 55 Incorporated, Yellow Springs, Ohio, 4387, USA). Moreover, temperature, electrical conductivity (EC), total dissolved solids (TDS) and salinity were recorded by Combo metre (H M Digital, Inc. CA 90230). These observations were recorded randomly from different replicates.

\section{Blood pH, carcass traits and serum mineral chemistry}

At the end of $42 \mathrm{~d}$, two birds were randomly selected from each replicate for carcass and blood responses (Tables 4 and 5). Blood was collected from wing vein in EDTAcoated vacutainer for immediate $\mathrm{pH}$ monitoring. The same birds were killed by severing neck vein and blood samples were collected. Blood serum was separated by centrifugation of blood samples at $2,000 \times \mathrm{g}$ for $15 \mathrm{~min}$ (Hayat et al., 1999; Ahmad et al., 2005) and was analyzed for minerals ( $\mathrm{Na}, \mathrm{K}, \mathrm{Cl}, \mathrm{Ca}, \mathrm{P}, \mathrm{Mg}, \mathrm{HCO}_{3}$, Table 6) contents. The same birds were further used for evaluation of carcass characteristics. Carcass responses were evaluated in terms of dressing, breast, thigh, abdominal fat, gizzard, proventriculus, heart, liver, kidney, spleen, pancreas, bursa, gallbladder, intestine and lung weights, and shank and intestine lengths and were presented as percent of dressing weight. 


\section{Statistical analysis}

The experiment was executed under completely randomized design with factorial arrangement using four (4) levels of dNa from two (2) sources of salt. The replicate was an experimental unit. The data obtained at the end of each phase was subjected to ANOVA technique using GLM of Minitab 16.1 (Minitab, 2010). While the data collected at the end of the experiment, two-way ANOVA with level of $\mathrm{dNa}$ and source of salt as fixed effects and their interactions was applied. Linear and quadratic effects were studied in

Table 2. i) Effect of dietary sodium and sodium salts on body weight gain, feed intake and gain:feed of broilers during various phases of the experiment (Continued)

\begin{tabular}{|c|c|c|c|c|c|c|}
\hline & & d 1 to 10 & d 11 to 20 & d 21 to 33 & d 34 to 42 & d 1 to 42 \\
\hline Main effects (dNa/Salt) & & - & ------------ 1 & weight gain & ) ------------ & --------- \\
\hline \multirow[t]{4}{*}{$\mathrm{dNa}(\mathrm{g} / \mathrm{kg})$} & 1.7 & 149 & 336 & 862 & 546 & 1,893 \\
\hline & 2.6 & 144 & 344 & 831 & 640 & 1,959 \\
\hline & 3.5 & 150 & 328 & 840 & 564 & 1,883 \\
\hline & 4.4 & 141 & 314 & 851 & 558 & 1,864 \\
\hline \multirow[t]{2}{*}{ Salt } & $\mathrm{NaHCO}_{3}$ & 137 & 317 & 838 & 591 & 1,884 \\
\hline & $\mathrm{Na}_{2} \mathrm{SO}_{4}$ & 155 & 344 & 854 & 563 & 1,916 \\
\hline \multicolumn{7}{|c|}{ Interaction effects (Salt×dNa) } \\
\hline $\mathrm{NaHCO}_{3}$ & 1.7 & 147 & 338 & 864 & 592 & 1,941 \\
\hline $\mathrm{NaHCO}_{3}$ & 2.6 & 138 & 324 & 849 & 630 & 1,941 \\
\hline $\mathrm{NaHCO}_{3}$ & 3.5 & 143 & 295 & 807 & 584 & 1,829 \\
\hline $\mathrm{NaHCO}_{3}$ & 4.4 & 122 & 311 & 833 & 558 & 1,824 \\
\hline $\mathrm{Na}_{2} \mathrm{SO}_{4}$ & 1.7 & 151 & 334 & 859 & 500 & 1,844 \\
\hline $\mathrm{Na}_{2} \mathrm{SO}_{4}$ & 2.6 & 150 & 364 & 813 & 650 & 1,977 \\
\hline $\mathrm{Na}_{2} \mathrm{SO}_{4}$ & 3.5 & 157 & 361 & 874 & 545 & 1,936 \\
\hline $\mathrm{Na}_{2} \mathrm{SO}_{4}$ & 4.4 & 161 & 318 & 868 & 558 & 1,905 \\
\hline SEM & & 2.0 & 9.0 & 18.0 & 27.3 & 34.1 \\
\hline \multicolumn{7}{|l|}{$\mathrm{p}$-value $(\mathrm{df}=24)$} \\
\hline & Level & 0.376 & 0.049 & 0.794 & 0.741 & 0.247 \\
\hline & Source & $\leq 0.001$ & 0.188 & 0.451 & 0.304 & 0.306 \\
\hline & Salt $\times \mathrm{dNa}$ & 0.008 & 0.635 & 0.220 & 0.369 & 0.036 \\
\hline & Level (L) & 0.713 & 0.428 & 0.316 & 0.069 & 0.176 \\
\hline & Level (Q) & 0.177 & 0.657 & 0.663 & 0.055 & 0.156 \\
\hline \multicolumn{2}{|l|}{ Main effects (dNa/Salt) } & & ----------- & intake $(\mathrm{g} / \mathrm{bir}$ & ------------ & ----------- \\
\hline \multirow[t]{4}{*}{$\mathrm{dNa}(\mathrm{g} / \mathrm{kg})$} & 1.7 & 235 & 643 & 1,649 & 1,036 & 3,564 \\
\hline & 2.6 & 227 & 663 & 1,654 & 1,089 & 3,634 \\
\hline & 3.5 & 234 & 652 & 1,652 & 1,067 & 3,605 \\
\hline & 4.4 & 228 & 681 & 1,612 & 1,101 & 3,622 \\
\hline \multirow[t]{2}{*}{ Salt } & $\mathrm{NaHCO}_{3}$ & 222 & 653 & 1,640 & 1,089 & 3,604 \\
\hline & $\mathrm{Na}_{2} \mathrm{SO}_{4}$ & 240 & 667 & 1,644 & 1,058 & 3,608 \\
\hline \multicolumn{7}{|c|}{ Interaction effects (Salt $\times \mathrm{dNa}$ ) } \\
\hline $\mathrm{NaHCO}_{3}$ & 1.7 & 236 & 635 & 1644 & 1,071 & 3,586 \\
\hline $\mathrm{NaHCO}_{3}$ & 2.6 & 222 & 658 & 1652 & 1,099 & 3,631 \\
\hline $\mathrm{NaHCO}_{3}$ & 3.5 & 216 & 650 & 1725 & 1,057 & 3,649 \\
\hline $\mathrm{NaHCO}_{3}$ & 4.4 & 215 & 668 & 1539 & 1,129 & 3,551 \\
\hline $\mathrm{Na}_{2} \mathrm{SO}_{4}$ & 1.7 & 234 & 651 & 1654 & 1,002 & 3,542 \\
\hline $\mathrm{Na}_{2} \mathrm{SO}_{4}$ & 2.6 & 232 & 669 & 1657 & 1,080 & 3,637 \\
\hline $\mathrm{Na}_{2} \mathrm{SO}_{4}$ & 3.5 & 251 & 653 & 1578 & 1,078 & 3,560 \\
\hline $\mathrm{Na}_{2} \mathrm{SO}_{4}$ & 4.4 & 242 & 694 & 1685 & 1,073 & 3,694 \\
\hline SEM & & 4.6 & 22.4 & 69.5 & 33.9 & 64.9 \\
\hline \multicolumn{7}{|l|}{$p$-value $(\mathrm{df}=24)$} \\
\hline & Level & 0.371 & 0.163 & 0.606 & 0.122 & 0.485 \\
\hline & Source & $\leq 0.001$ & 0.391 & 0.944 & 0.209 & 0.935 \\
\hline & Salt $\times \mathrm{dNa}$ & 0.001 & 0.880 & 0.569 & 0.727 & 0.267 \\
\hline & Level (L) & 0.692 & 0.776 & 0.651 & 0.692 & 0.572 \\
\hline & Level (Q) & 0.076 & 0.314 & 0.894 & 0.235 & 0.482 \\
\hline
\end{tabular}


Table 2. ii) Effect of dietary sodium and sodium salts on body weight gain, feed intake and gain:feed of broilers during various phases of experiment

\begin{tabular}{|c|c|c|c|c|c|c|}
\hline & & d 1 to 10 & d 11 to 20 & d 21 to 33 & d 34 to 42 & d 1 to 42 \\
\hline \multicolumn{7}{|c|}{ Main effects (dNa/salt) } \\
\hline \multirow[t]{4}{*}{$\mathrm{dNa}(\mathrm{g} / \mathrm{kg})$} & 1.7 & 1.58 & 1.94 & 1.92 & 2.06 & 1.95 \\
\hline & 2.6 & 1.59 & 1.92 & 2.00 & 1.71 & 1.80 \\
\hline & 3.5 & 1.57 & 2.01 & 1.97 & 1.90 & 1.89 \\
\hline & 4.4 & 1.64 & 2.22 & 1.90 & 1.98 & 1.95 \\
\hline \multirow[t]{2}{*}{ Salt } & $\mathrm{NaHCO}_{3}$ & 1.64 & 2.10 & 1.97 & 1.85 & 1.88 \\
\hline & $\mathrm{Na}_{2} \mathrm{SO}_{4}$ & 1.55 & 1.95 & 1.93 & 1.98 & 1.91 \\
\hline \multicolumn{7}{|c|}{ Interaction effects (Salt×dNa) } \\
\hline $\mathrm{NaHCO}_{3}$ & 1.7 & 1.61 & 1.88 & 1.91 & 1.81 & 1.82 \\
\hline $\mathrm{NaHCO}_{3}$ & 2.6 & 1.62 & 2.05 & 1.96 & 1.76 & 1.83 \\
\hline $\mathrm{NaHCO}_{3}$ & 3.5 & 1.54 & 2.22 & 2.14 & 1.81 & 1.91 \\
\hline $\mathrm{NaHCO}_{3}$ & 4.4 & 1.78 & 2.25 & 1.86 & 2.02 & 1.98 \\
\hline $\mathrm{Na}_{2} \mathrm{SO}_{4}$ & 1.7 & 1.55 & 1.96 & 1.93 & 2.32 & 2.08 \\
\hline $\mathrm{Na}_{2} \mathrm{SO}_{4}$ & 2.6 & 1.55 & 1.84 & 2.04 & 1.66 & 1.76 \\
\hline $\mathrm{Na}_{2} \mathrm{SO}_{4}$ & 3.5 & 1.61 & 1.81 & 1.81 & 1.99 & 1.88 \\
\hline $\mathrm{Na}_{2} \mathrm{SO}_{4}$ & 4.4 & 1.51 & 2.20 & 1.94 & 1.93 & 1.91 \\
\hline SEM & & 0.065 & 0.078 & 0.082 & 0.134 & 0.107 \\
\hline \multicolumn{7}{|c|}{$\mathrm{p}$-value $(\mathrm{df}=24)$} \\
\hline & Level & 0.371 & 0.035 & 0.763 & 0.925 & 0.792 \\
\hline & Source & 0.088 & 0.154 & 0.521 & 0.466 & 0.755 \\
\hline & Salt $\times \mathrm{dNa}$ & 0.231 & 0.505 & 0.672 & 0.315 & 0.172 \\
\hline & Level (L) & 0.497 & 0.349 & 0.183 & 0.211 & 0.189 \\
\hline & Level (Q) & 0.600 & 0.837 & 0.816 & 0.383 & 0.401 \\
\hline
\end{tabular}

$\mathrm{L}=$ Linear; $\mathrm{Q}=$ Quadratic. Number of observations per mean value $=40$ birds $\times 4$ replicates $=160$ birds.

the model by using polynomial contrasts. The level of significance was 0.05 unless or otherwise stated.

\section{RESULTS}

At the start of the experiment, the analysis of water was performed for sodium absorption (25.6) and residual sodium carbonate (9.02). The concentration of various minerals (cations plus anions) and values of $\mathrm{pH}$ (7.17 to 7.49), DO (3.70 to $5.60 \mathrm{mg} / \mathrm{L})$, temperature (24.0 to $29.7^{\circ} \mathrm{C}$ ), EC (1.06 to 1.39 millisiemens/cm), TDS (1,060 to $1,284 \mathrm{ppm})$ and salinity (1.2 to $1.3 \mathrm{~g} / \mathrm{kg})$ of the drinking water were also analyzed and found within the satisfactory range (detailed data not shown).

In the present experiment, the growth performance (BWG, FI, and FG) was affected by dNa levels, salts or their interaction (level $\times$ source) only during the first half (prestarter and starter) of the experiment and was not affected in the second half (grower and finisher) of the experiment. During d 1 to 10 , diets containing $\mathrm{Na}_{2} \mathrm{SO}_{4}$ salt (interaction effects) showed highest BWG $(\mathrm{p} \leq 0.008)$ and FI $(\mathrm{p} \leq 0.001)$ at 4.4 and $3.5 \mathrm{~g} / \mathrm{kg}$, respectively. The BWG $(\mathrm{p} \leq 0.049)$ and $\mathrm{FG}(\mathrm{p} \leq 0.035)$ were improved on $2.6 \mathrm{~g} / \mathrm{kg}$
dNa level during d 11 to 20 (Table 2). The supplementation of $\mathrm{Na}_{2} \mathrm{SO}_{4}$ to attain $2.6 \mathrm{~g} / \mathrm{kg} \mathrm{dNa}$ ameliorated BWG during the overall study period i.e. $d 1$ to 42 ( $\mathrm{p} \leq 0.036$; interaction effects). In the rest of the experimental phases, the $\mathrm{dNa}$ levels, salts or their interaction (level $\times$ source) was found not to influence any of the growth parameters. Mortality of birds among all the dietary treatments were found nonsignificant therefore were excluded from the tables.

A significant rise in DWI with increasing $\mathrm{dNa}$ levels was noticed during $\mathrm{d} 21$ to $33(\mathrm{p} \leq 0.007)$, d 34 to 42 $(p \leq 0.012)$ and $d \quad 1$ to $42 \quad(p \leq 0.036)$. The highest consumption of water was observed at the highest level of dNa i.e. $4.4 \mathrm{~g} / \mathrm{kg}$. The supplementation of both salts (main effect) or level $\times$ source (interaction effect) did not influence DWI during the overall experimental period. A higher value of DWI:FI was found in $\mathrm{NaHCO}_{3}$ supplemented diets during $\mathrm{d} 1$ to $10(\mathrm{p} \leq 0.036 ; 3.03)$ and in $\mathrm{Na}_{2} \mathrm{SO}_{4}$ supplemented diets during d 34 to 42 ( $\mathrm{p} \leq 0.026$; 2.51; Table $3)$. Both levels and sources of dNa did not affect DWI:FI during the rest of the phases. On the other hand, litter moisture contents and mortality were not affected by dNa, salt or interaction of both ( $p>0.05$; data not shown).

The increasing amount of $\mathrm{dNa}$ from either source (level 
Table 3. Effect of dietary sodium and sodium salts on water intake and water intake-to-feed intake ratio of broilers during various phases of experiment

\begin{tabular}{|c|c|c|c|c|c|c|}
\hline Item & & d 1 to 10 & d 11 to 20 & d 21 to 33 & d 34 to 42 & d 1 to 42 \\
\hline Main effects (dNa/salt) & & - & - & Water intake & -------------- & ------------ \\
\hline \multirow[t]{4}{*}{$\mathrm{dNa}(\mathrm{g} / \mathrm{kg})$} & 1.7 & 685 & 1,777 & 3,411 & 2,422 & 8,294 \\
\hline & 2.6 & 683 & 1,817 & 3,740 & 2,657 & 8,898 \\
\hline & 3.5 & 666 & 1,751 & 3,730 & 2,561 & 8,709 \\
\hline & 4.4 & 650 & 1,722 & 3,835 & 2,733 & 8,940 \\
\hline \multirow[t]{2}{*}{ Salts } & $\mathrm{NaHCO}_{3}$ & 674 & 1,766 & 3,589 & 2,544 & 8,574 \\
\hline & $\mathrm{Na}_{2} \mathrm{SO}_{4}$ & 668 & 1,767 & 3,769 & 2,642 & 8,846 \\
\hline \multicolumn{7}{|c|}{ Interaction effects (Salt×dNa) } \\
\hline $\mathrm{NaHCO}_{3}$ & 1.7 & 720 & 1,833 & 3,340 & 2,349 & 8,241 \\
\hline $\mathrm{NaHCO}_{3}$ & 2.6 & 695 & 1,740 & 3,651 & 2,596 & 8,683 \\
\hline $\mathrm{NaHCO}_{3}$ & 3.5 & 670 & 1,796 & 3,631 & 2,472 & 8,570 \\
\hline $\mathrm{NaHCO}_{3}$ & 4.4 & 611 & 1,696 & 3,734 & 2,760 & 8,801 \\
\hline $\mathrm{Na}_{2} \mathrm{SO}_{4}$ & 1.7 & 650 & 1,721 & 3,482 & 2,494 & 8,347 \\
\hline $\mathrm{Na}_{2} \mathrm{SO}_{4}$ & 2.6 & 671 & 1,894 & 3,829 & 2,718 & 9,112 \\
\hline $\mathrm{Na}_{2} \mathrm{SO}_{4}$ & 3.5 & 662 & 1,706 & 3,830 & 2,649 & 8,848 \\
\hline $\mathrm{Na}_{2} \mathrm{SO}_{4}$ & 4.4 & 689 & 1,747 & 3,936 & 2,706 & 9,078 \\
\hline SEM & & 36.9 & 77.4 & 86.0 & 97.1 & 109.1 \\
\hline \multicolumn{7}{|l|}{$\mathrm{p}$-value $(\mathrm{df}=24)$} \\
\hline & Level & 0.305 & 0.354 & 0.007 & 0.012 & 0.036 \\
\hline & Source & 0.821 & 0.988 & 0.073 & 0.168 & 0.135 \\
\hline & Salt $\times \mathrm{dNa}$ & 0.062 & 0.620 & 0.817 & 0.386 & 0.820 \\
\hline & Level (L) & 0.788 & 0.524 & 0.254 & 0.651 & 0.301 \\
\hline & Level (Q) & 0.896 & 0.564 & 0.301 & 0.063 & 0.137 \\
\hline \multicolumn{2}{|l|}{ Main effects (dNa/salt) } & -------- & ------- Water & e-to-feed int & tio $(\mathrm{mL} / \mathrm{g})-$ & ------ \\
\hline \multirow[t]{4}{*}{$\mathrm{dNa}(\mathrm{g} / \mathrm{kg})$} & 1.7 & 2.91 & 2.76 & 2.09 & 2.36 & 2.33 \\
\hline & 2.6 & 3.01 & 2.74 & 2.28 & 2.44 & 2.45 \\
\hline & 3.5 & 2.87 & 2.71 & 2.28 & 2.40 & 2.42 \\
\hline & 4.4 & 2.84 & 2.53 & 2.40 & 2.48 & 2.47 \\
\hline \multirow[t]{2}{*}{ Salts } & $\mathrm{NaHCO}_{3}$ & 3.03 & 2.72 & 2.20 & 2.34 & 2.38 \\
\hline & $\mathrm{Na}_{2} \mathrm{SO}_{4}$ & 2.79 & 2.66 & 2.32 & 2.51 & 2.46 \\
\hline \multicolumn{7}{|c|}{ Interaction effects (Salt×dNa) } \\
\hline $\mathrm{NaHCO}_{3}$ & 1.7 & 3.05 & 2.89 & 2.03 & 2.19 & 2.30 \\
\hline $\mathrm{NaHCO}_{3}$ & 2.6 & 3.13 & 2.64 & 2.22 & 2.36 & 2.39 \\
\hline $\mathrm{NaHCO}_{3}$ & 3.5 & 3.10 & 2.78 & 2.13 & 2.34 & 2.35 \\
\hline $\mathrm{NaHCO}_{3}$ & 4.4 & 2.84 & 2.55 & 2.43 & 2.44 & 2.48 \\
\hline $\mathrm{Na}_{2} \mathrm{SO}_{4}$ & 1.7 & 2.78 & 2.64 & 2.15 & 2.52 & 2.36 \\
\hline $\mathrm{Na}_{2} \mathrm{SO}_{4}$ & 2.6 & 2.90 & 2.84 & 2.34 & 2.52 & 2.51 \\
\hline $\mathrm{Na}_{2} \mathrm{SO}_{4}$ & 3.5 & 2.63 & 2.63 & 2.43 & 2.46 & 2.49 \\
\hline $\mathrm{Na}_{2} \mathrm{SO}_{4}$ & 4.4 & 2.84 & 2.51 & 2.36 & 2.53 & 2.46 \\
\hline SEM & & 0.106 & 0.128 & 0.123 & 0.102 & 0.087 \\
\hline \multicolumn{7}{|l|}{$\mathrm{p}$-value $(\mathrm{df}=24)$} \\
\hline & Level & 0.482 & 0.083 & 0.070 & 0.293 & 0.167 \\
\hline & Source & 0.036 & 0.517 & 0.296 & 0.028 & 0.243 \\
\hline & Salt $\times \mathrm{dNa}$ & 0.574 & 0.701 & 0.699 & 0.249 & 0.685 \\
\hline & Level (L) & 0.567 & 0.393 & 0.755 & 0.986 & 0.573 \\
\hline & Level (Q) & 0.472 & 0.762 & 0.546 & 0.438 & 0.397 \\
\hline
\end{tabular}

$\mathrm{L}=$ Linear; $\mathrm{Q}=$ Quadratic. Number of observations per mean value $=40$ birds $\times 4$ replicates $=160$ birds.

$\times$ source effects) were shown to rise blood $\mathrm{pH}\left(\mathrm{p} \leq 0.033\right.$; compared to $\mathrm{Na}_{2} \mathrm{SO}_{4}$ supplemented diets. In the present Table 4). The impact of $\mathrm{NaHCO}_{3}$ supplemented diets on study, dressing weight $(\mathrm{p} \leq 0.001)$ and abdominal fat blood $\mathrm{pH}$ at higher $\mathrm{dNa}$ levels is more pronounced as $(\mathrm{p} \leq 0.001$; quadratic effect) were reduced, whereas breast 
Table 4. Effect of dietary sodium and sodium salts on blood and carcass responses of broilers at the end of the experiment

\begin{tabular}{|c|c|c|c|c|c|c|c|}
\hline Item & & Blood pH & $\begin{array}{l}\text { Dressing } \\
\text { weight }^{1}\end{array}$ & Breast weight ${ }^{2}$ & Thigh weight ${ }^{2}$ & Abdominal fat ${ }^{1}$ & Lame birds $^{3}$ \\
\hline \multicolumn{8}{|c|}{ Main effects (dNa/salt) } \\
\hline \multirow[t]{4}{*}{$\mathrm{dNa}(\mathrm{g} / \mathrm{kg})$} & 1.7 & 7.30 & 56.12 & 31.85 & 45.26 & 3.01 & 6.2 \\
\hline & 2.6 & 7.33 & 54.18 & 32.98 & 46.28 & 3.12 & 3.1 \\
\hline & 3.5 & 7.35 & 52.94 & 33.79 & 47.34 & 2.44 & 3.8 \\
\hline & 4.4 & 7.37 & 52.07 & 34.31 & 48.30 & 2.61 & 4.1 \\
\hline \multirow[t]{2}{*}{ Salts } & $\mathrm{NaHCO}_{3}$ & 7.35 & 53.95 & 33.16 & 46.28 & 2.74 & 5.1 \\
\hline & $\mathrm{Na}_{2} \mathrm{SO}_{4}$ & 7.33 & 53.70 & 33.30 & 47.31 & 2.86 & 3.6 \\
\hline \multicolumn{8}{|c|}{ Interaction effects $($ Salt $\times \mathrm{dNa})$} \\
\hline $\mathrm{NaHCO}_{3}$ & 1.7 & 7.31 & 55.68 & 32.11 & 45.29 & 2.96 & 5.0 \\
\hline $\mathrm{NaHCO}_{3}$ & 2.6 & 7.34 & 54.85 & 32.56 & 45.05 & 2.93 & 4.5 \\
\hline $\mathrm{NaHCO}_{3}$ & 3.5 & 7.36 & 53.38 & 33.57 & 46.79 & 1.93 & 7.2 \\
\hline $\mathrm{NaHCO}_{3}$ & 4.4 & 7.38 & 51.90 & 34.40 & 48.01 & 3.12 & 3.5 \\
\hline $\mathrm{Na}_{2} \mathrm{SO}_{4}$ & 1.7 & 7.30 & 56.56 & 31.60 & 45.24 & 3.06 & 7.5 \\
\hline $\mathrm{Na}_{2} \mathrm{SO}_{4}$ & 2.6 & 7.32 & 53.50 & 33.40 & 47.51 & 3.31 & 1.8 \\
\hline $\mathrm{Na}_{2} \mathrm{SO}_{4}$ & 3.5 & 7.34 & 52.51 & 34.01 & 47.90 & 2.94 & 0.2 \\
\hline $\mathrm{Na}_{2} \mathrm{SO}_{4}$ & 4.4 & 7.36 & 52.24 & 34.22 & 48.58 & 2.11 & 4.8 \\
\hline SEM & & 0.003 & 0.599 & 0.492 & 0.886 & 0.35 & 1.76 \\
\hline \multicolumn{8}{|c|}{$\mathrm{p}$-value $(\mathrm{df}=56)$} \\
\hline & Level & $\leq 0.001$ & $\leq 0.001$ & $\leq 0.001$ & $\leq 0.001$ & $\leq 0.001$ & 0.312 \\
\hline & Source & 0.011 & 0.561 & 0.678 & 0.109 & 0.014 & 0.240 \\
\hline & Salt $\times \mathrm{dNa}$ & 0.033 & 0.770 & 0.853 & 0.927 & 0.915 & 0.048 \\
\hline & Level (L) & 0.001 & 0.212 & 0.385 & 0.960 & 0.567 & 0.173 \\
\hline & Level (Q) & - & 0.854 & 0.983 & 0.955 & 0.018 & 0.092 \\
\hline
\end{tabular}

${ }^{1} \%$ of live weight (without visceral organs). ${ }^{2} \%$ of dressed weight. ${ }^{3}$ Percentages.

$\mathrm{L}=$ Linear; $\mathrm{Q}=$ Quadratic. Number of observations per mean value $=2$ birds $\times 4$ replicates $=8$ birds

$(\mathrm{p} \leq 0.001)$ and thigh $(\mathrm{p}<0.001)$ weights were aggravated with increasing $\mathrm{dNa}$ (linear effects; Table 4). The level $\times$ source interaction influenced the number of lame birds $(\mathrm{p} \leq 0$. 048).

The weights of the gizzard $(\mathrm{p} \leq 0.002)$, bursa $(\mathrm{p} \leq 0.001$; quadratic effect), gall bladder $(\mathrm{p} \leq 0.003)$, lungs $(\mathrm{p} \leq 0.033$; linear effect) and intestine $(\mathrm{p} \leq 0.031$; quadratic effect) responded to $\mathrm{dNa}$ levels while the weights of the kidney $(\mathrm{p} \leq 0.001)$ and gall bladder $(\mathrm{p} \leq 0.001)$ were influenced by replacing salts (Table 5 ). The level $\times$ source interaction was found to influence weights of the kidney $(\mathrm{p} \leq 0.006)$, bursa $(p \leq 0.001)$ and intestine $(p \leq 0.001)$. The weights of the proventriculus, heart, liver, spleen and pancreas, and shank length were measured and found similar between all dietary treatments (data not shown; $\mathrm{p}>0.05$ ).

A quadratic increase in serum $\mathrm{Na}^{+}(\mathrm{p} \leq 0.002), \mathrm{K}^{+}, \mathrm{Cl}^{-}$ and $\mathrm{Mg}^{2+}(\mathrm{p} \leq 0.001)$, whereas a linear rise in serum $\mathrm{HCO}^{-}$ and $\mathrm{Ca}^{2+}$ were observed with increasing $\mathrm{dNa}$ from 1.7 to 4.4 $\mathrm{g} / \mathrm{kg}$ (Table 6).

\section{DISCUSSION}

The concentration of electrolytes and other attributes (EC, TDS, salinity) of water are anticipated to maneuver the electrolyte concentration of bird's digesta. The results obtained from the present study indicated that the electrolyte concentration in the water was too low to have any impact on the growth performance. The range of water pH i.e. 6.0 to 8.5 has been considered as optimal for broiler performance (Socha et al., 2002; Borges et al., 2003a, 2003b), whereas water TDS levels between 1,000 to 3,000 ppm were considered satisfactory for broilers (Chiba, 2009).

The present experiment was conducted to find out the exact requirements of $\mathrm{dNa}$ and its salt for each described phase of broiler's life but in most of the stances we remained uncertain to bring forth the amount of $\mathrm{dNa}$ for each phase. The improvement of BWG and FI during d 1 to 10 indicated that higher levels of sulphates in $\mathrm{Na}_{2} \mathrm{SO}_{4}$ supplemented diets might induce appetite in birds since better absorption of sulphates is $\mathrm{Na}$ dependent active process or because $\mathrm{Na}$ involves in cysteine sparing effect as per Langridge-Smith et al. (1983), Ahearn and Murer (1984) and Florin et al. (1991). But, this weight gain was not translated into better FG per se, which showed that birds ate and weighed more but did not convert feed into weight efficiently as it has to be. Afterwards, the amelioration in BWG and FG signified that during these crucial days of life broilers were more efficiently able to transform feed into weight per se. Among the growth 
Table 5. Effect of dietary sodium and sodium salts on body organ weights ${ }^{1}$ of broilers at the end of the experiment

\begin{tabular}{|c|c|c|c|c|c|c|c|}
\hline Item & & Gizzard & Kidney & Bursa & Gall bladder & Lungs & $\begin{array}{c}\text { Intestinal } \\
\text { weight }^{2}\end{array}$ \\
\hline Main effects (dNa/salt) & & ------ & 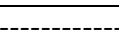 & $--\%$ of & sed weight ---. & --------- & -----------. \\
\hline \multirow[t]{4}{*}{$\mathrm{dNa}(\mathrm{g} / \mathrm{kg})$} & 1.7 & 2.41 & 0.31 & 0.23 & 0.08 & 0.56 & 58.2 \\
\hline & 2.6 & 2.57 & 0.36 & 0.15 & 0.08 & 0.58 & 54.3 \\
\hline & 3.5 & 2.98 & 0.37 & 0.21 & 0.09 & 0.60 & 54.9 \\
\hline & 4.4 & 2.99 & 0.37 & 0.25 & 0.12 & 0.48 & 56.1 \\
\hline \multirow[t]{2}{*}{ Salts } & $\mathrm{NaHCO}_{3}$ & 2.85 & 0.42 & 0.21 & 0.07 & 0.58 & 56.3 \\
\hline & $\mathrm{Na}_{2} \mathrm{SO}_{4}$ & 2.62 & 0.28 & 0.22 & 0.11 & 0.54 & 55.4 \\
\hline \multicolumn{8}{|c|}{ Interaction effects $($ Salt×dNa) } \\
\hline $\mathrm{NaHCO}_{3}$ & 1.7 & 2.72 & 0.42 & 0.16 & 0.07 & 0.64 & 58.8 \\
\hline $\mathrm{NaHCO}_{3}$ & 2.6 & 2.65 & 0.47 & 0.18 & 0.07 & 0.61 & 56.2 \\
\hline $\mathrm{NaHCO}_{3}$ & 3.5 & 2.98 & 0.41 & 0.22 & 0.04 & 0.54 & 58.7 \\
\hline $\mathrm{NaHCO}_{3}$ & 4.4 & 3.05 & 0.39 & 0.26 & 0.11 & 0.51 & 51.6 \\
\hline $\mathrm{Na}_{2} \mathrm{SO}_{4}$ & 1.7 & 2.11 & 0.21 & 0.29 & 0.10 & 0.49 & 57.7 \\
\hline $\mathrm{Na}_{2} \mathrm{SO}_{4}$ & 2.6 & 2.48 & 0.24 & 0.13 & 0.09 & 0.56 & 52.4 \\
\hline $\mathrm{Na}_{2} \mathrm{SO}_{4}$ & 3.5 & 2.98 & 0.33 & 0.20 & 0.13 & 0.64 & 51.1 \\
\hline $\mathrm{Na}_{2} \mathrm{SO}_{4}$ & 4.4 & 2.93 & 0.35 & 0.24 & 0.12 & 0.46 & 60.7 \\
\hline SEM & & 0.21 & 0.04 & 0.02 & 0.01 & 0.04 & 1.62 \\
\hline \multicolumn{8}{|l|}{$\mathrm{p}$-value $(\mathrm{df}=56)$} \\
\hline & Level & 0.002 & 0.089 & 0.294 & 0.003 & 0.089 & 0.321 \\
\hline & Source & 0.408 & $\leq 0.001$ & 0.614 & $\leq 0.001$ & 0.218 & 0.346 \\
\hline & Salt $\times \mathrm{dNa}$ & 0.379 & 0.006 & $\leq 0.001$ & 0.575 & 0.099 & $\leq 0.001$ \\
\hline & Level (L) & 0.682 & 0.403 & 0.109 & 0.184 & 0.033 & 0.101 \\
\hline & Level (Q) & 0.169 & 0.873 & $\leq 0.001$ & 0.972 & 0.420 & 0.031 \\
\hline
\end{tabular}

${ }^{1}$ Weights of proventriculus, heart, liver, and pancreas, and shank length were excluded from the data because of having non-significant effects.

${ }^{2}$ Measured in grams.

$\mathrm{L}=$ Linear; $\mathrm{Q}=$ Quadratic. Number of observations per mean value $=2$ birds $\times 4$ replicates $=8$ birds .

Table 6. Effect of dietary sodium and sodium salts on serum mineral chemistry of broilers at the end of the experiment

\begin{tabular}{|c|c|c|c|c|c|c|c|}
\hline \multicolumn{2}{|l|}{ Item } & $\mathrm{Na}$ & $\mathrm{K}$ & $\mathrm{Cl}$ & $\mathrm{Ca}$ & $\mathrm{Mg}$ & $\mathrm{HCO}_{3}$ \\
\hline \multicolumn{2}{|c|}{ Main effects (dNa/salt) } & \multicolumn{6}{|c|}{ 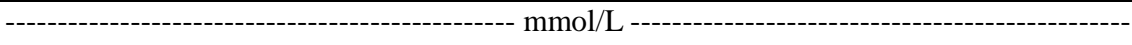 } \\
\hline \multirow[t]{4}{*}{$\mathrm{dNa}(\mathrm{g} / \mathrm{kg})$} & 1.7 & 131 & 3.04 & 102 & 2.52 & 1.06 & 30.8 \\
\hline & 2.6 & 132 & 3.05 & 102 & 2.56 & 1.15 & 31.1 \\
\hline & 3.5 & 135 & 3.07 & 104 & 2.68 & 1.18 & 30.0 \\
\hline & 4.4 & 137 & 3.04 & 101 & 2.71 & 1.14 & 30.6 \\
\hline \multirow[t]{2}{*}{ Salts } & $\mathrm{NaHCO}_{3}$ & 134 & 3.06 & 102 & 2.50 & 1.14 & 31.4 \\
\hline & $\mathrm{Na}_{2} \mathrm{SO}_{4}$ & 132 & 3.05 & 102 & 2.73 & 1.12 & 30.8 \\
\hline \multicolumn{8}{|c|}{ Interaction effects (Salt×dNa) } \\
\hline $\mathrm{NaHCO}_{3}$ & 1.7 & 132 & 3.04 & 103 & 2.45 & 1.11 & 31.1 \\
\hline $\mathrm{NaHCO}_{3}$ & 2.6 & 134 & 3.06 & 102 & 2.40 & 1.07 & 31.4 \\
\hline $\mathrm{NaHCO}_{3}$ & 3.5 & 137 & 3.09 & 104 & 2.56 & 1.28 & 29.7 \\
\hline $\mathrm{NaHCO}_{3}$ & 4.4 & 138 & 3.02 & 100 & 2.60 & 1.11 & 30.7 \\
\hline $\mathrm{Na}_{2} \mathrm{SO}_{4}$ & 1.7 & 130 & 3.04 & 101 & 2.58 & 1.01 & 30.5 \\
\hline $\mathrm{Na}_{2} \mathrm{SO}_{4}$ & 2.6 & 131 & 3.04 & 102 & 2.71 & 1.22 & 30.7 \\
\hline $\mathrm{Na}_{2} \mathrm{SO}_{4}$ & 3.5 & 133 & 3.05 & 103 & 2.79 & 1.09 & 30.2 \\
\hline $\mathrm{Na}_{2} \mathrm{SO}_{4}$ & 4.4 & 135 & 3.06 & 102 & 2.82 & 1.18 & 30.4 \\
\hline SEM & & 0.3 & 0.005 & 0.4 & 0.079 & 0.025 & 0.205 \\
\hline \multicolumn{8}{|l|}{ p-value } \\
\hline & Level & $<0.001$ & 0.001 & 0.001 & 0.005 & 0.001 & 0.011 \\
\hline & Source & 0.231 & 0.031 & 0.431 & $<0.001$ & 0.292 & 0.324 \\
\hline & Salt $\times \mathrm{dNa}$ & 0.121 & 0.097 & 0.543 & 0.089 & 0.077 & 0.223 \\
\hline & Level (L) & $<0.001$ & 0.001 & 0.001 & 0.007 & $<0.001$ & 0.007 \\
\hline & Level (Q) & 0.002 & $<0.001$ & $<0.001$ & 0.064 & $<0.001$ & 0.019 \\
\hline
\end{tabular}

$\mathrm{L}=$ Linear; $\mathrm{Q}=$ Quadratic. Number of observations per mean value $=2$ birds $\times 4$ replicates $=8$ birds. 
parameters it seems that BWG is more sensitive to the level and source of $\mathrm{dNa}$. It is clear from the present results that selection of right dietary salt and $\mathrm{dNa}$ level could play a crucial role particularly in feed intake (prestarter) and feed conversion (starter) and birds are more sensitive to the level and source of $\mathrm{Na}$ during these days rather when they become heavier, however birds showed the direct impact of $\mathrm{dNa}$ on body weight throughout their life and the requirements of $\mathrm{dNa}$ were decreased over the age.

With regards to the increasing DWI with increasing $\mathrm{dNa}$ levels during the whole experimental period, the findings of Julian et al. (1992), Murakami et al. (2000) and Rondon et al. (2001) also confirmed the enhanced water consumption with increasing level of $\mathrm{dNa}$. It is anticipated that higher level of dNa could modify body fluids and osmotic balance that increases thirst and leads to increased water consumption (Smith and Teeter, 1989; Borges et al., 2004a, 2004b). This water is not excreted through faeces in the present study as evident from the similar litter condition ( $p>0.05$ ) at all levels of $\mathrm{dNa}$ and is thought to retain in the muscle tissues, which is also reflected from higher dressing weight observed in the present study.

It is suggested that the higher water consumption limits the gut capacity for feed intake and excretes nutrients in faeces thus reduces weight as evident from lower BWG in $\mathrm{NaHCO}_{3}$ and $\mathrm{Na}_{2} \mathrm{SO}_{4}$ supplemental diets during first and last ten days of age, respectively, in the present study. These results are in contrast with Hooge et al. (1999) who reported that added level of $\mathrm{dNa}$ from $\mathrm{Na}_{2} \mathrm{SO}_{4}$ didn't affect DWI:FI and litter moisture while he noticed moderate effects on these parameters when $\mathrm{NaHCO}_{3}$ was used to add dNa. The contrary results might be due the impact of a number of coccidiostats used by Hooge et al. (1999) that changed the water intake behavior in birds.

Patience et al. (1986) stated that metabolizable ions like $\mathrm{HCO}_{3}{ }^{-}$cause acid neutralization and increase blood $\mathrm{pH}$. In our study, the increased $\mathrm{pH}$ in $\mathrm{NaHCO}_{3}$ supplemental diets resulted in the numerical decrease in BWG at $\mathrm{d} 42$ ( $\mathrm{p}>0.05)$. The value of $\mathrm{pH}$ greater than 7.35 is known to reduce growth performance in broilers (Ahmad et al., 2005) as evident from the numerical decline in weight gain with increasing levels of dNa. Mushtaq et al. (2007) also observed rise in $\mathrm{pH}$ by increasing $\mathrm{dNa}(2.0$ to $3.0 \mathrm{~g} / \mathrm{kg}$ ) during d 29 to 42 . The decline in weight gain in the present study might be the impact of acid base imbalance. Increased levels of $\mathrm{dNa}$ should be compensated with $\mathrm{dCl}$ so as to conserve nominal effects of alkalosis that is the main ideology behind electrolyte balance theory. As per Lehninger (1970), for proper functioning of intermediary metabolism and cellular activity, body physiological system requires an optimum $\mathrm{pH}$, which in turn controls enzymatic activity and ultimately leads towards better growth performance. According to the statement of Mongin (1981) when DEB is more or less than $250 \mathrm{mEq} / \mathrm{kg}$ of diet, it either causes alkalosis or acidosis that lead to retarded growth performance. This is also evident from the present study that highest level of $\mathrm{dNa}(4.4 \mathrm{~g} / \mathrm{kg} ; \mathrm{DEB}=320 \mathrm{mEq} / \mathrm{kg})$ showed poor growth performance $(1,864 \mathrm{~g})$ as compared to $2.6 \mathrm{~g} / \mathrm{kg} \mathrm{dNa}$ or DEB $=240 \mathrm{mEq} / \mathrm{kg}(1,959 \mathrm{~g}$; $\mathrm{p}>0.05)$.

The higher breast and thigh meat yield in the present study might be attributed to increased water accumulation in muscle tissues as higher water intake $(\mathrm{p} \leq 0.04)$ was observed in high dNa diets. The findings of Borgatti et al. (2004) are in line with our results who also observed higher weights of breast and thigh meat with increasing level of DEB. Contrarily, Mushtaq et al. (2007) observed reduced breast and leg meat by increasing dNa from 2.0 to $3.0 \mathrm{~g} / \mathrm{kg}$. These attributes (breast and thigh meat weights) were negatively affected by heat stress condition provided in the experiment by Mushtaq et al. (2007) therefore it is obvious that more nutrients were utilized to maintain acid base balance and not converted into meat. It can, therefore, be suggested from the present study that by increasing $\mathrm{dNa}$ under normal physiological condition might improve the basal metabolism, which leads to proper energy utilization for meat production and thus very less energy is wasted for abdominal fat deposition.

The increase in weight and size of most of the body organs (i.e. hypertrophy) is the indication of stress condition (Koong et al., 1985), which might be induced by high levels of $\mathrm{dNa}$. The higher weight of lymphoid organ (bursa) showed intruded immune function of the body in the present study and might result in numerically retarded growth performance. In case of other organs, Johnson and Karunajeewa (1985), Borges et al. (1999) and Borgatti et al. (2004) also did not observe any combination effects of electrolytes on the weights of proventriculus, heart, liver, and pancreas, and shank length.

A direct response of $\mathrm{dNa}$ was noticed in the concentration of serum $\mathrm{Na}^{+}$and the explanation of increased level of serum $\mathrm{K}^{+}$and $\mathrm{Cl}^{-}$might be to compensate higher level of serum $\mathrm{Na}^{+}$and to sustain acid base balance. Mushtaq et al. (2005) suggested that cations and anions maintain each other in the body fluids in order to keep the acid-base homeostasis. This homeostasis could be maintained by providing proper DEB so that mild alkalosis caused by increased dNa could be compensated by dietary supplementation of anions. Further, it was observed that serum $\mathrm{Na}^{+}$and $\mathrm{K}^{+}$are directly related to each other in the blood and leads towards osmoregulation of body fluids (Mushtaq et al., 2007). An elevated level of serum $\mathrm{HCO}_{3}{ }^{-}$is the direct response of dietary $\mathrm{NaHCO}_{3}$ that caused an increase in $\mathrm{pH}$, which resulted in retarded growth parameters as reflected in the present experiment. Ahmad et al. (2005) suggested $\mathrm{NaHCO}_{3}$ as the best choice among salts. The contradiction among results might be the 
difference in heat stress condition, which causes metabolic acidosis and increased the requirement of $\mathrm{HCO}_{3}{ }^{-}$.

\section{CONCLUSION}

It can be inferred from the present findings that higher dietary sodium $(2.6$ to $3.5 \mathrm{~g} / \mathrm{kg}$ ) from sodium sulphate is the better choice in broiler diets for better growth efficiency especially in the first half (prestarter and starter) of life. The weight gain and feed intake could be optimized by reducing dietary sodium and water intake from d 21 to 42 (grower and finisher). The breast and thigh meat yield could be improved by increasing dietary sodium. Moreover, it is suggested to verify the requirements of dietary sodium by changing other electrolytes (potassium and chloride) with same or other salt sources at constant dietary electrolyte balance.

\section{ACKNOWLEDGEMENTS}

The present experiment was supported and sponsored by the Research Model Broiler Unit, Poultry Research and Training Center, Department of Poultry Production, University of Veterinary and Animal Sciences, Ravi Campus, Lahore, Pakistan. Funding for chemical analyses of diet and blood was provided by Higher Education Commission (HEC), Islamabad, Pakistan under the program 'Indigenous PhD Fellowship Program (5000 Fellowships)' Batch-V. Special thanks to the students of BS (Hons) Poultry Science (Batch 2007-2011) for their contribution in data collection. The contribution of Zulfiqar Ali (ExNutritional Consultant, Big Feeds (Pvt) Ltd, Lahore, Pakistan) in the experimental diet formulation, preparation and analyses is highly appreciated. The authors are highly grateful to Rural Development Administration, Republic of Korea for paying the publication charges of the manuscript.

\section{REFERENCES}

Ahearn, G. A. and H. Murer. 1984. Functional roles of $\mathrm{Na}^{+}$and $\mathrm{H}^{+}$ in $\mathrm{SO}_{4}{ }^{2-}$ transport by rabbit ileal brush border membrane vesicles. J. Membr. Biol. 78:177-186.

Ahmad, T., M. Sarwar, Mahr-un-Nisa, Ahsan-ul-Haq, and Zia-ulHasan. 2005. Influence of varying sources of dietary electrolytes on the performance of broilers reared in a high temperature environment. Anim. Feed Sci. Technol. 120:277298.

Ahmad, T., T. Mushtaq, M. A. Khan, M. E. Babar, M. Yousaf, Ziaul-Hassan, and Z. Kamran. 2009. Influence of varying dietary electrolyte balance on broiler performance under tropical summer conditions. J. Anim. Physiol. Anim. Nutr. 93:613-621.

AOAC. 2005. Official methods of analysis. 18th edn. Association of Official Analytical Chemists, Gaithersburg, Maryland.

Borgatti, L. M. O., R. Albuquerque, N. C. Meister, L. W. O. Souza, F. R. Lima, and M. A. Trindade Neto. 2004. Performance of broilers fed diets with different dietary electrolyte balance under summer conditions. Rev. Bras. Cienc. Avic. 6:153-157.

Borges, S. A., J. Ariki, C. L. Martins, and V. M. B. deMoraes. 1999. Potassium chloride supplementation in heat stressed broilers. Rev. Bras. Zootec. 28:313-319.

Borges, S. A., A. V. Fischer da Silva, J. Ariki, D. M. Hooge, and K. R. Cummings. 2003a. Dietary electrolyte balance for broiler chickens under moderately high ambient temperatures and relative humidities. Poult. Sci. 82:301-308.

Borges, S. A., A. V. Fischer da Silva, J. Ariki, D. M. Hooge, and K. R. Cummings. 2003b. Dietary electrolyte balance for broiler chickens exposed to thermoneutral or heat-stress environments. Poult. Sci. 82:482-435.

Borges, S. A., A. V. Fisher da Silva, A. Majorka, D. M. Hooge, and K. R. Cummings. 2004a. Physiological responses of broiler chickens to heat stress and dietary electrolyte balance (sodium plus potassium minus chloride, milliequivalents per kilogram). Poult. Sci. 83:1551-1558.

Borges, S. A., A. V. Fisher da Silva, A. D. A. Meira, T. Moura, A. Maiorka, and A. Ostrensky. 2004b. Electrolyte balance in broiler growing diets. Int. J. Poult. Sci. 3:623-628.

Chiba, L. I. 2009. Water and electrolytes (and Iodine). In: Animal Nutrition Handbook. 2nd rev. edn. Auburn Univ. Press, Alabama, USA.

Degussa AG Feed Additives. 2006. AminoDat 3.0. The Amino Acid Composition of Feedstuffs, Platinum Version. 6th rev. ed. Degussa AG Feed Additives, Hanau Wolfgang, Germany.

Florin, T., G. Neale, G. R. Gibson, and J. H. Cummings. 1991. Metabolism of dietary sulphate: Absorption and excretion in humans. Gut 32:766-773.

Gorman, I. and D. Balnave. 1994. Effects of dietary mineral supplementation on the performance and mineral excretions of broilers at high ambient temperatures. Br. Poult. Sci. 35:563572.

Han, Y. and D. H. Baker. 1994. Digestible lysine requirement of male and female broiler chicks during the period three to six weeks post-hatching. Poult. Sci. 73:1739-1745.

Hayat, J., D. Balnave, and J. Brake. 1999. Sodium bicarbonate and potassium supplements for broilers can cause poor performance at high temperature. Br. Poult. Sci. 40:411-418.

Hooge, D. M., K. R. Cummings, and J. L. McNaughton. 1999. Evaluation of sodium bicarbonate, chloride, or sulfate with a coccidiostat in corn-soy or corn-soy-meat diets for broiler chickens. Poult. Sci. 78:1300-1306.

Hubbard. 2004. Broiler Management Guide. Hubbard LLC, Duluth, Georgia.

Johnson, R. J. and H. Karunajeewa. 1985. The effects of dietary minerals and electrolytes on the growth and physiology of the young chick. J. Nutr. 115:1680-1690.

Julian, R. J., L. J. Caston, and S. Leeson. 1992. The effect of dietary sodium on right ventricular failure-induced ascites, gain and fat deposition in meat-type chickens. Can. J. Vet. Res. 56:214-219.

Koong, L. J., C. C. Farcell, and S. A. Nienata. 1985. Assessment of interrelationship among levels of intake and production, organ size and fasting heat production in animals. J. Nutr. 115:1383-1390.

Lacroix, R. L., D. R. Keeney, and L. M. Welsh. 1970. Potentiometric titration of chloride in plant tissue extracts 
using the chloride ion electrode. Commun. Soil Sci. Plant Anal. $1: 1-6$.

Langridge-Smith, J. E., J. H. Sellin, and M. Field. 1983. Sulphate influx across the rabbit ileal brush border membrane: Sodium and proton dependence, and substrate specificities. J. Membr. Biol. 72:131-139.

Leeson, S. and J. D. Summers. 2001. Scott's nutrition of the chicken. 4th Ed. University Books, Guelph, Ontario, Canada.

Lehninger, A. L. 1970. Biochemistry. Worth Publishers Inc., New York. pp. 50-51.

Minitab. 2010. Minitab, version 16.1.0. Minitab Inc., State College, Pennsylvania, USA.

Mongin, P. 1980. Electrolytes in nutrition. A review of basic principles and practical applications in poultry and swine. In: Proceedings of 3rd Annual International Minerals Conference, Orlando, FL. pp. 1.

Mongin, P. 1981. Recent advances in dietary cation-anion balance: Applications in poultry. In: Proceedings of the Nutrition Society. 40:285-294.

Murakami, A. E., E. A. Saleh, S. E. Watkins, and P. W. Waldroup. 2000. Sodium source and level in broiler diets with and without high levels of animal protein. J. Appl. Poult. Res. 9:53-61.

Murakami, A. E., E. O. O. Rondon., E. N. Martins, M. S. Pereira, and C. Scapinello. 2001. Sodium and chloride requirements of growing broiler chickens (twenty-one to forty-two days of age) fed corn-soybean diets. Poult. Sci. 80:289-294.

Mushtaq, T., M. Sarwar, H. Nawaz, M. A. Mirza, and T. Ahmad. 2005. Effect and interactions of dietary sodium and chloride on broiler starter performance (hatching to twenty-eight days of age) under subtropical summer conditions. Poult. Sci. 84:17161722 .
Mushtaq, T., M. A. Mirza, M. Athar, D. M. Hooge, T. Ahmad, G. Ahmad, M. M. H. Mushtaq, and U. Noreen. 2007. Dietary sodium and chloride for twenty-nine to forty-two-day-old broiler chickens at constant electrolyte balance under subtropical summer conditions. J. Appl. Poult. Res.16:161-170. Mushtaq, M. M. H., T. N. Pasha, M. Akram, T. Mushtaq, R. Parvin, H. C. Choi, J. Hwangbo, and J. H. Kim. 2013. Growth performance, carcass characteristics and plasma mineral chemistry as affected by dietary chloride and chloride salts fed to broiler chickens reared under phase feeding system. Asian Australas. J. Anim. Sci. 26:845-855.

National Research Council. 1994. Nutrient requirements of poultry. 9th Rev. Ed., National Academy Press, Washington, DC.

Patience, J. F., R. E. Austic, and R. D. Boyd. 1986. The effect of sodium bicarbonate or potassium bicarbonate on acid base status and protein and energy digestibility in swine. Nutr. Res. 6:263-273.

Rondon, E. O. O., A. E. Murakami, A. C. Furlan, I. Moreira, and M. Macari. 2001. Sodium and chloride requirements of young broiler chickens fed corn-soybean diets (one to twenty-one days of age). Poult. Sci. 80:592-598.

Ruiz-Lopez, B. and R. E. Austic. 1993. The effects of selected minerals on acid-base balance of growing chicks. Poult. Sci. 72:1054-1062.

Smith, M. O. and R. G. Teeter. 1989. Effects of sodium and potassium salts on gain, water consumption and body temperature of 4 to 7 week old heat stressed broilers. Nutr. Rep. Int. 40:161-169.

Socha, M. T., S. M. Ensley, D. J. Tomlinson, and B. Johnson. 2002. Variability of water consumption and potential impact on animal performance. In: Proceedings of California Animal Nutrition Conference, Fresno, CA. pp. 81-91. 